\title{
Fatty acid transport protein-2 regulates glycemic control and diabetic kidney disease progression
}

\author{
Shenaz Khan, ${ }^{1}$ Robert Gaivin, ${ }^{1}$ Caroline Abramovich, ${ }^{2}$ Michael Boylan, ${ }^{3}$ Jorge Calles, ${ }^{4}$ \\ and Jeffrey R. Schelling ${ }^{1,5}$ \\ 'Department of Medicine, Division of Nephrology, ${ }^{2}$ Department of Pathology, ${ }^{3}$ Department of Medicine, Division of \\ Gastroenterology, ${ }^{4}$ Department of Medicine, Division of Endocrinology, MetroHealth Campus, and ${ }^{5}$ Department of \\ Physiology and Biophysics, Case Western Reserve University, Cleveland, Ohio, USA.
}

\begin{abstract}
Kidney disease is one of the most devastating complications of diabetes, and tubular atrophy predicts diabetic kidney disease (DKD) progression to end-stage renal disease. We have proposed that fatty acids bound to albumin contribute to tubular atrophy by inducing lipotoxicity, after filtration across damaged glomeruli, and subsequent proximal tubule reabsorption by a fatty acid transport protein-2-dependent (FATP2-dependent) mechanism. To address this possibility, genetic (Lepr ${ }^{d b / d b}$ eNOS ${ }^{-/}$) and induced (high-fat diet plus low-dose streptozotocin) mouse models of obesity and DKD were bred with global FATP2 gene-deleted mice (SIc27a2) and then phenotyped. DKD-prone mice with the SIc27a2-/- genotype demonstrated normalization of glomerular filtration rate, reduced albuminuria, improved kidney histopathology, and longer life span compared with diabetic SIc27a2 $2^{+/+}$mice. Genetic and induced DKD-prone SIc27a2 ${ }^{-/-}$ mice also exhibited markedly reduced fasting plasma glucose, with mean values approaching euglycemia, despite increased obesity and decreased physical activity. Glucose lowering in DKD-

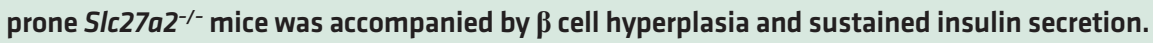
Together, our data indicate that FATP2 regulates DKD pathogenesis by a combined lipotoxicity and glucotoxicity (glucolipotoxicity) mechanism.
\end{abstract}

Conflict of interest: The authors have declared that no conflict of interest exists.

Copyright: () 2020, American Society for Clinical Investigation.

Submitted: January 29, 2020

Accepted: June 24, 2020

Published: August 6, 2020.

Reference information: /CI Insight. 2020;5(15):e136845.

https://doi.org/10.1172/jci.

insight.136845.

\section{Introduction}

Diabetes affects more than 30 million people in the United States (https://www.diabetes.org/resources/ statistics/statistics-about-diabetes) and is a major public health problem due to morbidity and mortality associated with end-organ complications. One of the most devastating complications, diabetic kidney disease (DKD), affects as many as 1 in 3 people with diabetes. DKD from type 2 diabetes is by far the most common cause of end-stage renal disease (1), which is only treatable with dialysis or kidney transplantation. Blood pressure and glucose control are important interventions to slow DKD progression but neither is sufficient to extinguish diabetic complications (2-7), emphasizing that the pathophysiology is incompletely understood and other factors are required.

Of many DKD biomarkers that have been tested, tubular atrophy and interstitial fibrosis are among the most reliable predictors of progression to end-stage renal disease (8). There are many inducible and genetic mouse models of type 2 diabetes, but few exhibit both the histologic and the functional features of DKD. Streptozotocin (STZ) has commonly been used to induce type 1 diabetes in rodents, but high doses of STZ may inflict tubular toxicity (9). However, low-dose STZ combined with a second hit, such as high-fat diet (HFD), mimics type 2 diabetes and DKD while avoiding the direct nephrotoxic effects (9-11). Lepr ${ }^{d b / d b}$ $e N O S^{-1}$ mice also faithfully phenocopy type 2 diabetes and $\mathrm{DKD}$, with decreased glomerular filtration rate (GFR), increased albuminuria, and histologic lesions that include glomerulosclerosis, tubular atrophy due to apoptosis, interstitial fibrosis, and proximal tubule cytoplasmic lipid droplets $(12,13)$.

The increased proximal tubule cytosolic fat content in DKD has led to the hypothesis that tubular atrophy and progressive kidney failure are due to lipotoxicity (13-15). Increases in both proximal tubule epithelial cell fatty acid synthesis and uptake have been implicated in DKD pathogenesis (16-18). Lipotoxicity due to enhanced proximal tubule fatty acid uptake is particularly relevant in DKD accompanied 
by albuminuria, which is associated with high risk of progression to end-stage renal disease. In this context, circulating albumin-bound fatty acids cross the injured glomerular filtration barrier, and downstream tubules are then exposed to the filtered fatty acids.

Fatty acid uptake is achieved through transport by a family of evolutionarily conserved fatty acid transport proteins (FATPs) (19). The most abundant FATP in kidney is FATP2 (encoded by the Slc27a2 gene), which is expressed predominately on the apical proximal tubule membrane (20-22). FATP2-dependent fatty acid uptake leads to apoptosis in proximal tubule cells in vitro (21), consistent with lipotoxicity as a mechanism of tubular atrophy. FATP2 inhibition has also been shown to have a modest glucose-lowering effect (23), which could be relevant to DKD pathogenesis by limiting glucotoxicity (24).

To test the impact of FATP2 on diabetes and DKD in vivo, we crossed genetic ( Lepr $^{d b / d b}$ eNOS $^{-/}$) and induced (HFD plus low-dose STZ) mouse models of obesity, type 2 diabetes, and DKD with global Slc27a2 $2^{-1-}$ mice. FATP2 deletion in both DKD models resulted in improved renal function and histopathology as well as markedly improved glycemic control, suggesting that FATP2 regulates DKD by glucolipotoxicity, the combined effect of proximal tubule lipotoxicity and glucotoxicity mechanisms.

\section{Results}

FATP2 gene deletion rescues DKD pathology. Mice were genotyped by PCR as described in Methods, and representative gels for each allele (Lepr, eNOS, and Slc27a2) are shown in Supplemental Figure 1 A-C (supplemental material available online with this article; https://doi.org/10.1172/jci.insight.136845DS1). FATP2 is expressed most prominently in the kidney and liver (19), and within the kidney, in the apical proximal tubule membrane (Supplemental Figure 2) (21). To test the effect of FATP2 on DKD progression, global Slc27a2-1 mice, which have no overt phenotype (25), were crossed with mice prone to develop DKD. Histopathology was compared between age-matched, congenic Slc27a2 $2^{+/+} \operatorname{Lepr}^{\mathrm{db} / \mathrm{db}} \operatorname{eNOS}^{+/+}$(phenocopies type 2 diabetes, but

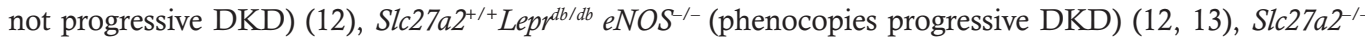
$\mathrm{Lepr}^{\mathrm{db} / \mathrm{db}} \mathrm{eNOS}^{-/-}$(experimental group), and Slc27a2 ${ }^{+/+} \mathrm{Lepr}^{+/+} \mathrm{eNOS}^{+/+}$(WT) mice.

Masson's trichrome-stained sections, which reveal more prominent interstitial fibrosis and fibro-

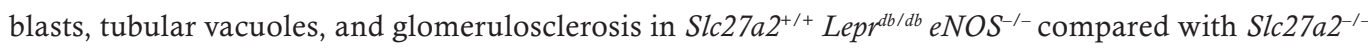
$\operatorname{Lepr}^{d b / d b} e \mathrm{NOS}^{-/-}$kidneys, are shown in Figure 1, A-I. Oil Red O-stained lipid droplets, which were

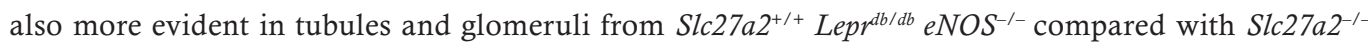
$\mathrm{Lepr}^{d b / d b} \mathrm{eNOS}^{-/-}$kidneys, are shown in Figure 1, J-O. Histologic data were analyzed by quantitative morphometry and revealed significant tubular atrophy, interstitial fibrosis, and glomerulosclerosis in

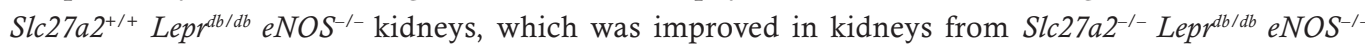
mice (Figure 1, P and Q). Proximal tubule apoptosis is a mechanism of tubular atrophy in mouse models of diabetic and nondiabetic kidney diseases (26). Figure $1 \mathrm{R}$ reveals increased apoptosis in

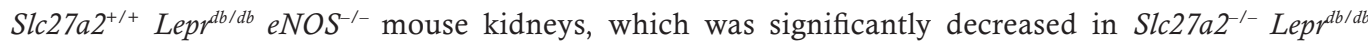
$e \mathrm{NOS}^{-/-}$kidneys. Inflammation has been implicated in DKD pathogenesis (27). IHC screening for macrophage infiltration revealed no significant difference between $S l c 27 a 2^{+/+}$Lepr $^{d b / d b} \mathrm{eNOS}^{-/-}$and Slc27a2 $2^{-/-}$ Lepr $^{d b / d b}$ eNOS $^{-/-}$kidneys (Supplemental Figure 3).

We then examined the effect of FATP2 deletion in a nongenetic model of type 2 diabetes and DKD, which was induced with HFD plus low-dose STZ. Figure 2 shows that glomerulosclerosis, tubular atrophy, and interstitial fibrosis were also less prominent in HFD plus STZ Slc27a2 ${ }^{-1-}$ kidneys. Taken together, the data in Figures 1 and 2 indicate that renal pathology in 2 heterogeneous models of DKD was ameliorated by deletion of the FATP2 gene.

A parsimonious explanation for the pathogenesis of tubular atrophy and interstitial fibrosis is epithelial-mesenchymal transition (EMT) (28), though fate tracing studies have cast doubt about this mechanism (29). To investigate whether tubular atrophy may be caused by fatty acid-induced EMT, human proximal tubule cells in culture were incubated with palmitate and screened for expression of mesenchymal markers, $\alpha$-smooth muscle actin and heat shock protein-47 (HSP47). Supplemental Figure 4 reveals no effect of palmitate on the expression of either mesenchymal marker, suggesting that fatty acids do not cause EMT, as a mechanism of tubular atrophy.

FATP2 gene deletion rescues renal function in DKD. To assess the effect of FATP2 on kidney function,

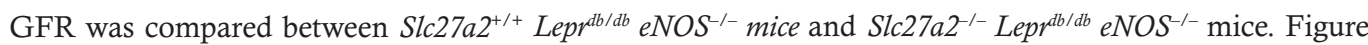

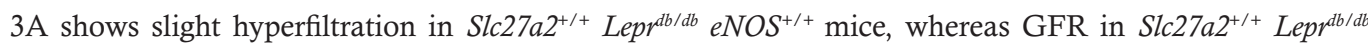
$\mathrm{eNOS}^{-/-}$was significantly decreased, consistent with prior reports (12). Importantly, mean GFR was normal 

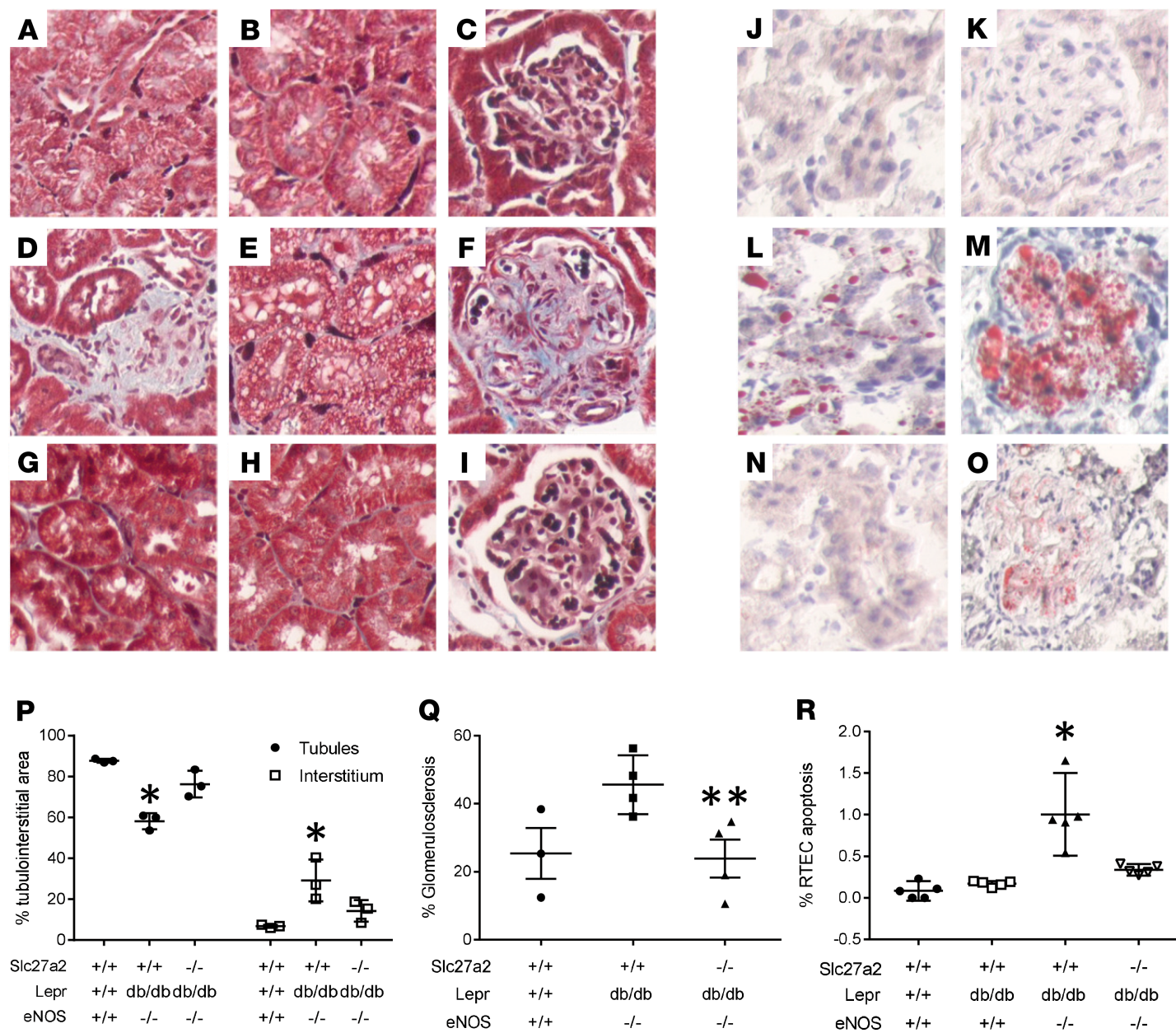

Figure 1. FATP2 gene deletion rescues pathology in a genetic DKD model. Masson's trichrome stain in WT (A-C), Slc27a2 $2^{+/+}$Lepr $^{\mathrm{db} / \mathrm{db}}$ eNOS $^{-1-}$ (D-F), and Slc27a2 ${ }^{-/-}$Lepr $^{\mathrm{db} / \mathrm{db}} \mathrm{eNOS}^{-I_{-}^{-}}$(G-I) kidneys. Oil Red 0 staining in frozen section from WT tubulointerstitium (J) and glomerulus (K), Slc27a2 ${ }^{+/+}$Lepr $^{\mathrm{db} / \mathrm{db}}$ eNOS $^{-1-}$ $(\mathbf{L}$ and $\mathbf{M})$, and SIc27a2 $2^{-/-}$Lepr $^{\mathrm{db} / \mathrm{db}} \mathrm{eNOS}^{-1-}(\mathbf{N}$ and $\mathbf{0})$ kidneys. Original magnification, $\times 600$. Quantitative histomorphometry to depict tubular atrophy and interstitial fibrosis (P) and glomerulosclerosis (Q). (R) Proximal tubule apoptosis by TUNEL assay. ${ }^{*}<0.001$ compared with other groups by ANOVA and Tukey's multiple comparisons test. ${ }^{* *} P=0.05$ compared with Slc27a2 ${ }^{+/+}$Lepr $^{\mathrm{db} / \mathrm{db}}$ eNOS ${ }^{-/-}$group by ANOVA and Tukey's multiple comparisons test. FATP2, fatty acid transport protein-2; DKD, diabetic kidney disease.

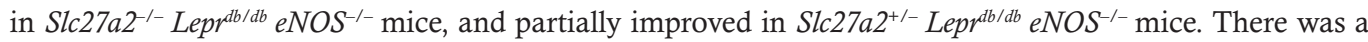
linear relationship between GFR and Slc27a2 null allele number in $\operatorname{Lepr}^{d b / d b} \mathrm{eNOS}^{-/-}$mice $\left(\mathrm{R}^{2}=0.98\right)$, and the difference in slope from zero approached statistical significance $(P=0.09$, Supplemental Figure 5), suggesting a FATP2 gene dose effect on GFR.

In WT mice fed a HFD for 6 months, baseline GFR was decreased compared with mice on a normal chow diet (compare Figure 3A with Figure 3B), a finding which has been previously described $(10,11)$. More importantly, GFR was significantly lower in the HFD plus low-dose STZ group and normalized in the HFD plus STZ-treated Slc27a2-/- mice (Figure 3B).

An alternative measure of renal function in $\mathrm{DKD}$ is albuminuria, which reflects a combination of glomerular filtration barrier and tubular dysfunction, and is commonly employed as a biomarker for DKD progression. Urine albumin/creatinine ratios were increased in the genetic (Figure 4A) and induced (Fig-

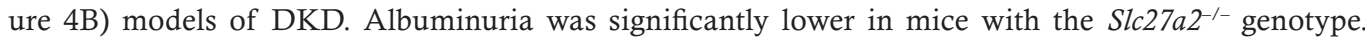
There was a modest inverse correlation between albuminuria and GFR in each model, though neither was statistically significant (Supplemental Figure 6). The data in Figures 3 and 4 data indicate that deletion of FATP2 ameliorated renal function in 2 different mouse models of type 2 diabetes and DKD.

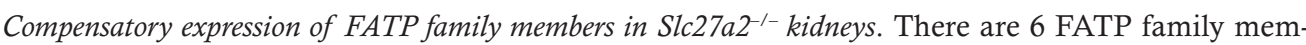
bers, and in WT kidneys, baseline expression of FATP2 is much greater than that of other FATP family

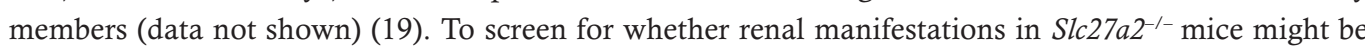



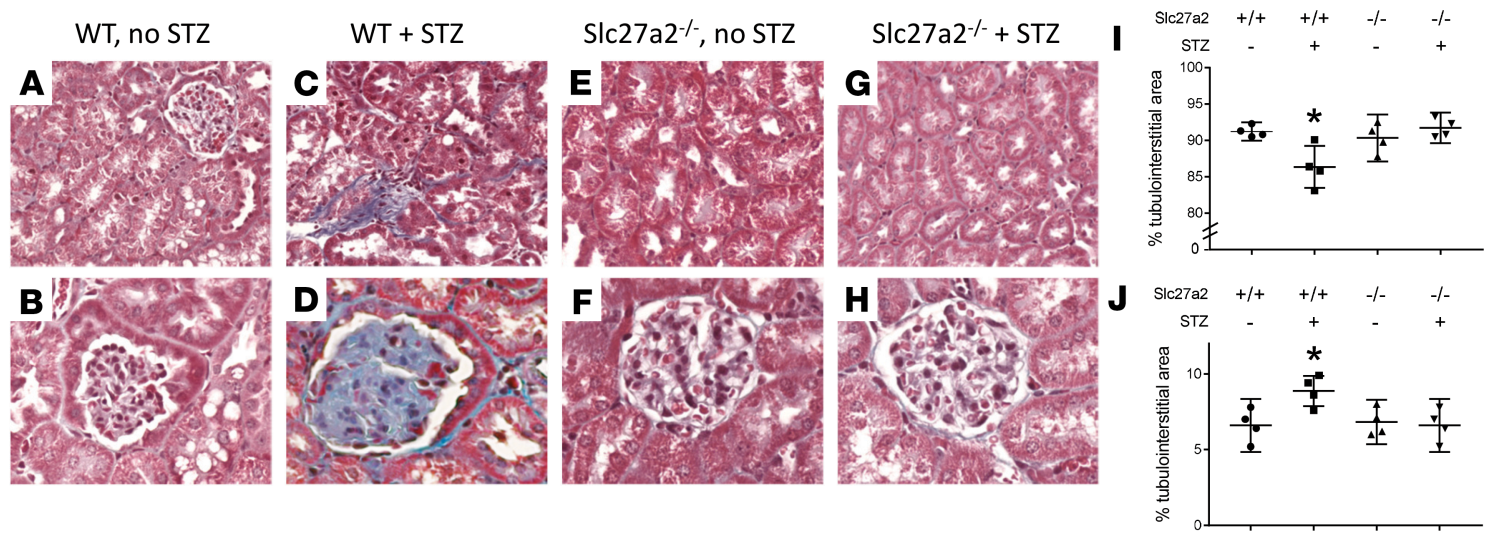

Figure 2. FATP2 gene deletion rescues pathology in an inducible DKD model. (A-H) Masson's trichrome stain in kidneys from WT and SIc27a2-1- mice fed HFD for 6 months with or without low-dose streptozotocin at 3 months, as described in Methods. Original magnification, $\times 200$ (top), $\times 400$ (bottom). Quantitative histomorphometry for tubular atrophy (I) and interstitial fibrosis (J) for each genotype. ${ }^{*} P<0.05$ compared with other 3 groups by ANOVA and Tukey's test for multiple comparisons. FATP2, fatty acid transport protein-2; DKD, diabetic kidney disease; HFD, high-fat diet.

attributable to other fatty acid transporters, we cataloged mRNA expression of all FATPs from Slc27a2-1kidneys by quantitative RT-PCR. Supplemental Figure 7 demonstrates that of the 6 FATP family members, the mRNA expression of FATP1, FATP3, FATP5, and FATP6 is increased by less than 1-fold in Slc27a2 ${ }^{-1-}$ mice. These data indicate that FATP2 gene deletion, rather than compensatory FATP expression, explains the renal phenotype rescue observed in the genetic and induced DKD models.

FATP2 deletion regulates glycemic control. Because Slc27a2-- mice are a global knockout, we next investigated the impact of extrarenal FATP2 on DKD. Hyperglycemia and glucotoxicity are critical to DKD pathogenesis (24), and fatty acid metabolism is intimately linked with glycemic control. We therefore tested fasting glucose levels in DKD mice harboring WT and FATP2 knockout alleles. Figure 5A reveals that,

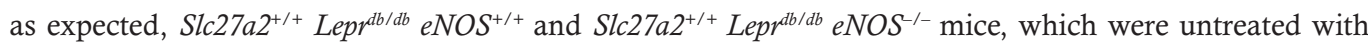
insulin, had elevated plasma glucose concentrations. Hyperglycemia, which often exceeded the upper limits of detectability ( $600 \mathrm{mg} / \mathrm{dL}$ ), was noted as early as 2 months of age (Supplemental Figure 8). The mean

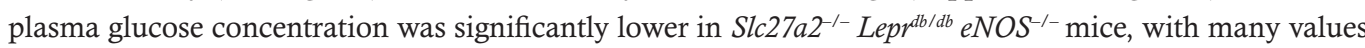
in the normal range. Intermediate plasma glucose values were observed in $\mathrm{Slc}^{2} \mathrm{Ta2^{+/- }} \mathrm{Lepr}^{\mathrm{db} / \mathrm{db}} \mathrm{eNOS}^{-/-}$mice, again suggesting a FATP2 gene dose effect. There was a linear relationship between plasma glucose and Slc27a2 null allele number in Lepr $r^{\text {db } / d b}$ eNOS $^{-/-}$mice $\left(\mathrm{R}^{2}=0.996\right)$, and the difference in slope from zero was

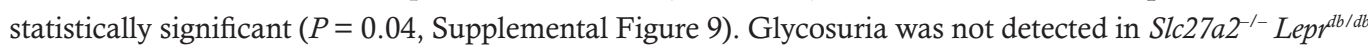
$e \mathrm{NOS}^{-1-}$ mice with plasma glucose less than $200 \mathrm{mg} / \mathrm{dL}$ (data not shown), indicating that enhanced urinary glucose excretion is not the mechanism of improved glycemic control.

In WT HFD plus STZ-treated mice, fasting plasma glucose was also elevated, but the magnitude was not as great as in Lepr didab mice (Figure 5B). Of note, the mean plasma glucose concentrations were significantly lower in the HFD plus STZ Slc27a2-- compared with the HFD plus STZ WT groups. Collectively, the data in Figure 5 raise the possibility that DKD pathogenesis may be due to glucolipotoxicity - the effect of both FATP2-regulated glucotoxicity and proximal tubule lipotoxicity.

A modest inverse correlation was noted between plasma glucose and GFR in the genetic model of DKD (Supplemental Figure 10A). Conversely, a stronger and positive correlation was observed between glucose and GFR in the inducible DKD model (Supplemental Figure 10B).

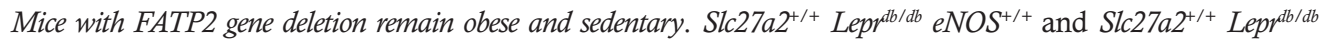

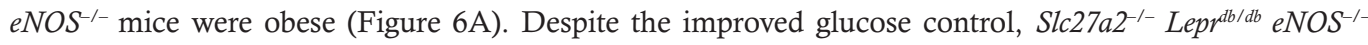
mice were super obese, with body weights that were routinely twice as great as WT littermates (Figure 6A). Slc27a2 ${ }^{-1-} \mathrm{Lepr}^{\mathrm{db} / \mathrm{db}} \mathrm{eNOS}^{-/-}$mice also consumed more chow (Figure 6B) and were less active (Figure 6C) compared with other groups. These data demonstrate that exercise and weight reduction were not mecha-

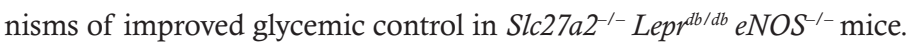

FATP2 deletion does not alter hepatosteatosis. Hepatic FATP2 is robustly expressed (Figure 7) and has been implicated in glucose metabolism (23). Furthermore, liver dysfunction due to hepatosteatosis is associated with insulin resistance and glucose intolerance (30). We therefore examined the effect of FATP2 
A

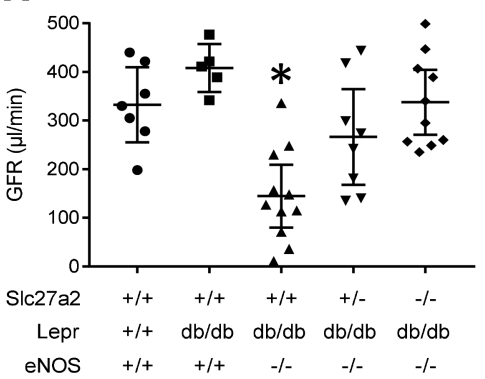

B

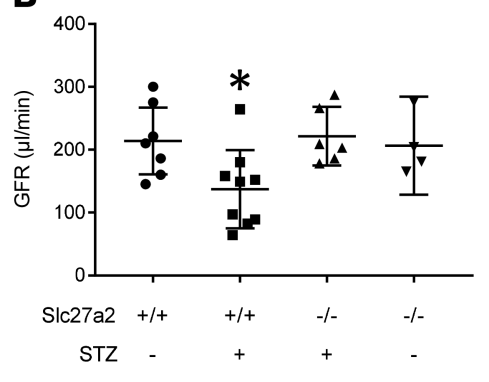

Figure 3. FATP2 gene deletion rescues GFR in DKD. (A) GFR was calculated by FITC-inulin decay, as described in Methods, in 5-month-old mice with indicated genotypes. ${ }^{*} P<0.005$ compared with all groups except Slc27a2 ${ }^{+1-}$ Lepr $^{\mathrm{db} / \mathrm{db}} \mathrm{eNOS}^{-1-}$. (B) GFR in 6-month-old mice with indicated genotypes on high-fat diets with or without low-dose streptozotocin (STZ). ${ }^{*} P=0.04$ compared with STZ-treated, SIc27a2-1- group. All statistical analyses by ANOVA and Tukey's test for multiple comparisons. FATP2, fatty acid transport protein-2; DKD, diabetic kidney disease; GFR, glomerular filtration rate; STZ, streptozotocin.

gene deletion on hepatosteatosis. Supplemental Figure 11A demonstrates modest steatosis scores in liv-

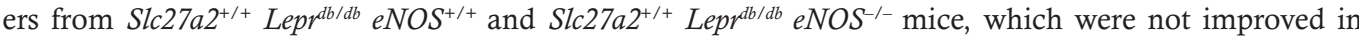

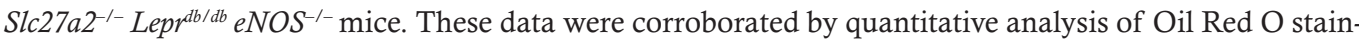
ing of triacylglycerol-containing lipid droplets (Supplemental Figure 11B). Although the cytoplasmic area

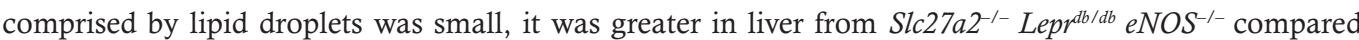

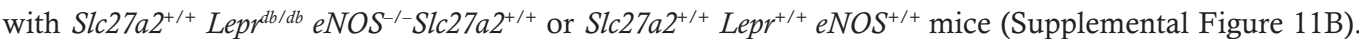

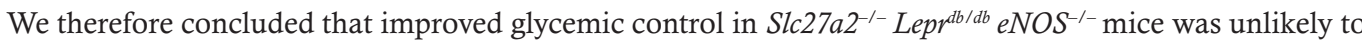
be mediated by liver FATP2 deletion.

FATP2 deletion preserves plasma insulin levels. FATP2 expression is most abundant in kidney and liver (19), though expression in other glucose-sensitive tissues has not been extensively investigated. As demonstrated in Figure 7, A and B, FATP2 mRNA was also expressed in heart, hypothalamus, small intestine, and pancreas. Because FATP2 mRNA was detected in pancreas, we next examined FATP2 effects on plasma insulin levels.

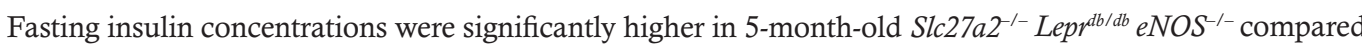

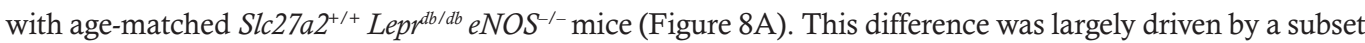
of mice with very high insulin levels, suggesting that despite improved glycemic control, many Slc27a2-1 $\mathrm{Lepr}^{\text {db/da }} \mathrm{eNOS}^{-1-}$ mice still developed insulin resistance. Indeed, Figure 8B demonstrates the interdependence and inverse relationship between fasting plasma glucose and insulin. In kinetic experiments, we observed

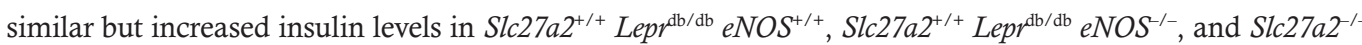
Lepr ${ }^{d b / d b} e \mathrm{NOS}^{-/-}$mice at 6 weeks (Figure 8C). At 3 and 6 months of age, insulin levels decreased markedly in

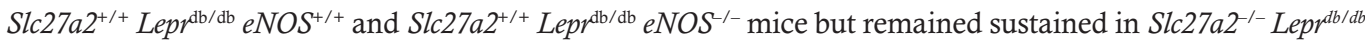
$\mathrm{eNOS}^{-/-}$mice, which corresponded with the plasma glucose differences between these groups.
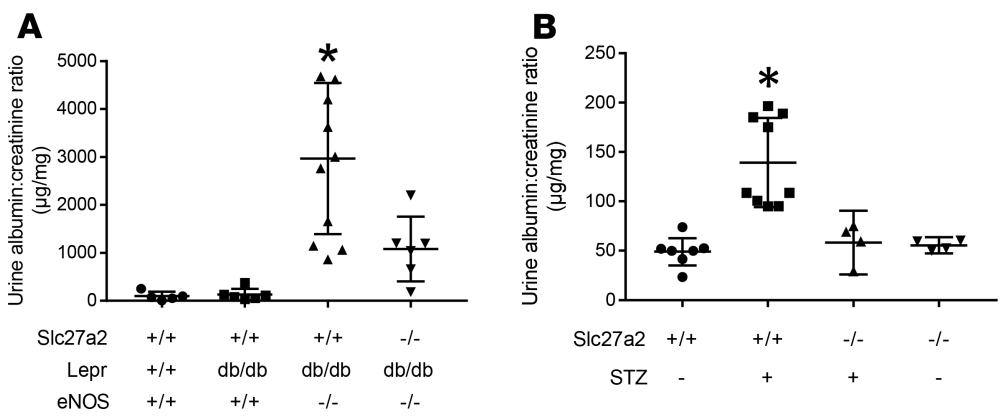

Figure 4. FATP2 gene deletion rescues albuminuria in DKD. (A) Urine albumin to creatinine ratios were determined by ELISA and colorimetric assays, respectively, as described in Methods. ${ }^{*} P<0.05$ compared with other groups by ANOVA and Tukey's test for multiple comparisons. (B) Albuminuria in 6-month-old mice with indicated genotypes on high-fat diets with or without low-dose streptozotocin. ${ }^{*} P<0.005$ compared with other groups by ANOVA and Tukey's test for multiple comparisons. FATP2, fatty acid transport protein-2; DKD, diabetic kidney disease. 
A

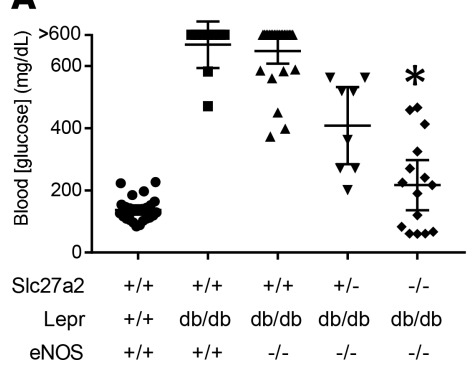

B

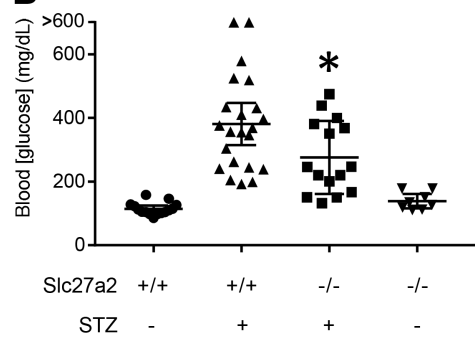

Figure 5. FATP2 deletion regulates glycemic control. (A) Fasting whole blood glucose was assayed in 5-month-old mice by glucometer from tail blood samples. ${ }^{*} P<0.001$ compared with Slc27a2 ${ }^{+/+}$Lepr $^{\mathrm{db} / \mathrm{db}} \mathrm{eNOS}^{-/-}$group by ANOVA and Tukey's test for multiple comparisons. (B) Blood glucose in 6-month-old mice with indicated Slc27a2 genotypes on high-fat diets with or without low-dose STZ. $P=0.03$ compared with SIc27a2 ${ }^{+/+}$plus STZ group by ANOVA and Tukey's test for multiple comparisons. FATP2, fatty acid transport protein-2; STZ, streptozotocin.

In WT mice fed a HFD, insulin levels were increased (Figure 8D), although this group remained euglycemic (Figure 5B). Mice on a HFD treated with low-dose STZ had significantly lower insulin levels, consistent with coincident hyperglycemia. Importantly, Slc27a2-- mice on a HFD with or without STZ maintained normal insulin levels, which was reflected by improved glycemic control. Taken together, these data indicate that in both the genetic and the inducible DKD models, FATP2 gene deletion prevented insulin insufficiency, resulting in lower plasma glucose concentrations.

FATP2 deletion is associated with pancreatic islet hypertrophy. Pancreas histology in 6-month-old Slc27a2+/+ $L e p r^{\mathrm{d} b / \mathrm{db}} e \mathrm{NOS}^{-1}$ mice demonstrated small islet size, with relatively few $\beta$ cells, prominent fibrosis, and

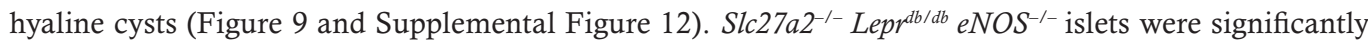

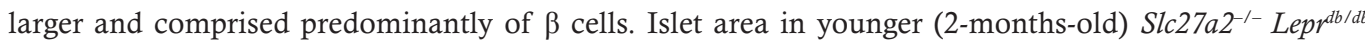
$e N O S^{-/-}$mice $\left(21,916 \pm 2,187 \mu \mathrm{m}^{2}\right)$ was $50 \%$ smaller than that in 6-month-old mice, and $\beta$ cells were positive for Ki-67 staining (Supplemental Figure 12, B-D, undetectable in other genotypes [data not shown]), indicating islet hypertrophy due to $\beta$ cell hyperplasia over time.

Mice on a HFD had significantly increased islet area (Supplemental Figure 13), consistent with observed hyperinsulinemia. In mice with inducible DKD from HFD plus low-dose STZ, FATP2 gene deletion was associated with a $46 \%$ increase in pancreatic islet area. Collectively, our data demonstrate that significantly lower plasma glucose concentrations in the genetic and inducible Slc27a2 ${ }^{--}$mouse models of DKD are due to $\beta$ cell hyperplasia and preserved insulin secretion.

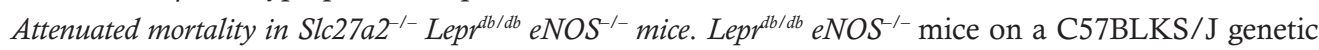
background have a reduced life span, and generally die before 26 weeks of age $(12,31)$, though mean survival rates as long as 9 months have been described $(26,32)$. Consistent with these reports, Figure 10

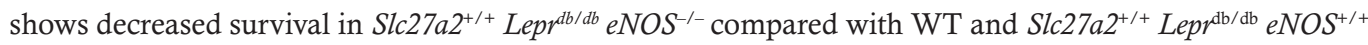

A

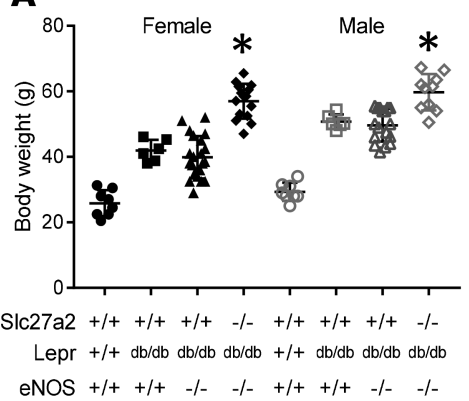

B

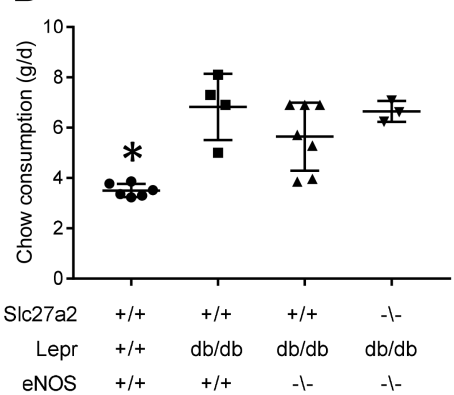

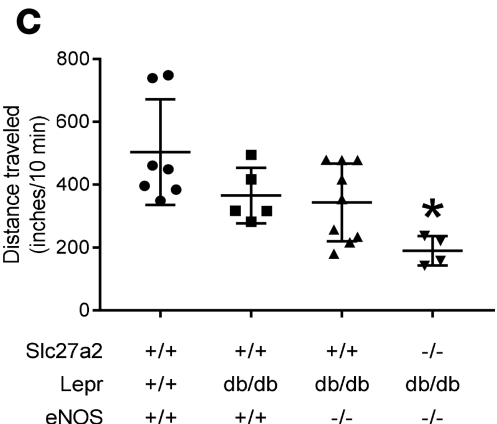

C

eNOS +/+ +/+ $-/-\quad-/-$

Figure 6. Diabetes-prone FATP2-knockout mice are persistently obese, hyperphagic, and sedentary. (A) Mean weekly body weights in 5-month-old mice with indicated gender and genotypes. ${ }^{*} P<0.001$ compared with other groups. (B) Mean daily chow consumption in 5 -month-old mice. ${ }^{*} P<0.01$ compared with other groups. (C) Physical activity, as determined by distance traveled over 10 minutes by videography, as described in Methods. $P=0.03$ compared with WT. All statistical analyses by ANOVA and Tukey's test for multiple comparisons. FATP2, fatty acid transport protein-2. 
A

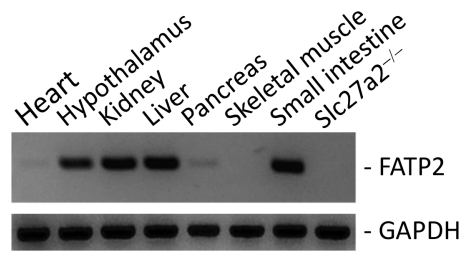

B

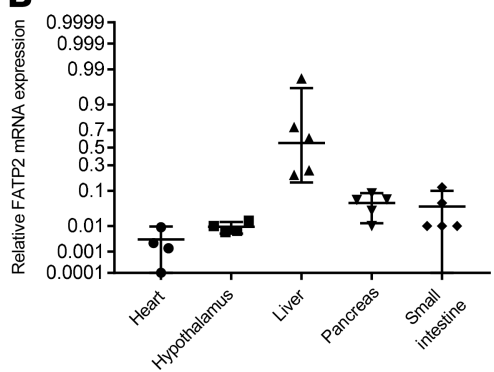

Figure 7. Tissue screen of FATP2 mRNA expression. (A) Representative gel after 30 cycles RT-PCR. Slc27a2 ${ }^{-l-}$ kid- $^{-}$ ney was used as a negative control. (B) Quantitative RT-PCR for FATP2 in tissues with most prominent expression. Data are normalized to kidney FATP2 expression, which is defined as 1.00 on the nonlinear $y$ axis. FATP2, fatty acid transport protein-2.

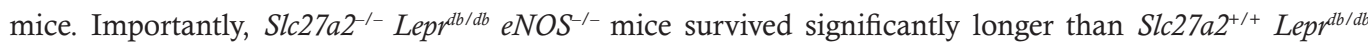
$e \mathrm{NOS}^{-/-}$mice. Necropsy findings revealed grossly normal organs and histologic evidence for kidney and liver disease as well as mild cardiac fibrosis in some Slc27a2 ${ }^{+/+}$Lepr $^{\text {db } / d b} \mathrm{eNOS}^{-/-}$mice (data not shown), but a definitive cause of death was not established.

\section{Discussion}

Lipids and lipid metabolites accumulate in tubules from humans and animal models of CKD, suggesting that lipotoxicity contributes to CKD pathogenesis $(14,15,24)$. Potential mechanisms include enhanced fatty acid uptake and synthesis as well as decreased utilization $(16,21,24,33)$. We tested the effect of FATP2-mediated fatty acid uptake in DKD pathogenesis using genetic (Lepr $\left.{ }^{\mathrm{db} / \mathrm{db}} e N O S^{--}\right)$and acquired (HFD plus low-dose STZ) mouse models of DKD with Slc27a2 deletion. We discovered that the Slc27a2 $2^{-1}$ genotype improved renal function and histology, glucose control, and mortality (summarized in Supplemental Table 1).

The major finding in this report is that FATP2 gene deletion prevented DKD progression, as defined by histology, GFR, and albuminuria. This is significant because state-of-the-art treatments in humans merely slow but do not halt DKD (2-6), suggesting that therapeutic strategies directed against additional, biologically diverse targets may be fruitful. Clinical evidence to support a role for renal lipotoxicity includes reports that treatment with the PPAR- $\alpha$ agonist fenofibrate, which promotes fatty acid utilization, slowed the rate of eGFR decline and albuminuria in DKD cohorts $(34,35)$. However, fenofibrate has not been widely adopted due to initial decreases in eGFR.

Resistance to tubular insults is likely to be important for preservation of kidney function, as evidenced by the association of sustained tubular injury with glomerulosclerosis and GFR decline $(8,36)$. Because FATP2 is localized predominately to the apical proximal tubule membrane in kidney (21), we have hypothesized that amelioration of renal function in FATP2-knockout mice would result from inhibited reabsorption of aberrantly filtered albumin-bound fatty acids, thereby limiting the combined impact of fatty acid uptake from the apical and basolateral surfaces. Indeed, mice prone to DKD with the $S l c 27 a 2^{--}$genotype derived significant protection against tubular atrophy and GFR reduction. Mouse models of calcineurin inhibitor and bisphosphonate toxicity demonstrated increased proximal tubule FATP2 expression, which was associated with accumulation of lipid droplets, interstitial fibrosis, and tubular epithelial cell apoptosis $(37,38)$. Furthermore, FATP2-knockout mice were resistant to bisphosphonate toxicity, similar to our data with DKD (38). Results from these animal models are consistent with in vitro data, which demonstrate that FATP2-dependent palmitate uptake causes apoptosis in multiple epithelial cell lines $(21,39)$. The biochemical mechanisms leading to lipoapoptosis are incompletely defined, but possibilities include impaired phospholipid-protein interaction, protein lipid modification, ceramide accumulation, endoplasmic reticulum stress, mitochondrial dysfunction, and reactive oxygen species generation $(13,40)$.

FATP2 deletion resulted in a near-complete reversal of the DKD phenotype, suggesting that FATP2 is the major fatty acid transporter regulating kidney glucolipotoxicity. These data are congruent with a paradigm whereby apical proximal tubule FATP2 serves as a scavenger receptor, to reclaim small amounts of filtered fatty acids under normal circumstances. In the context of glomerular damage, FATP2 participates in 

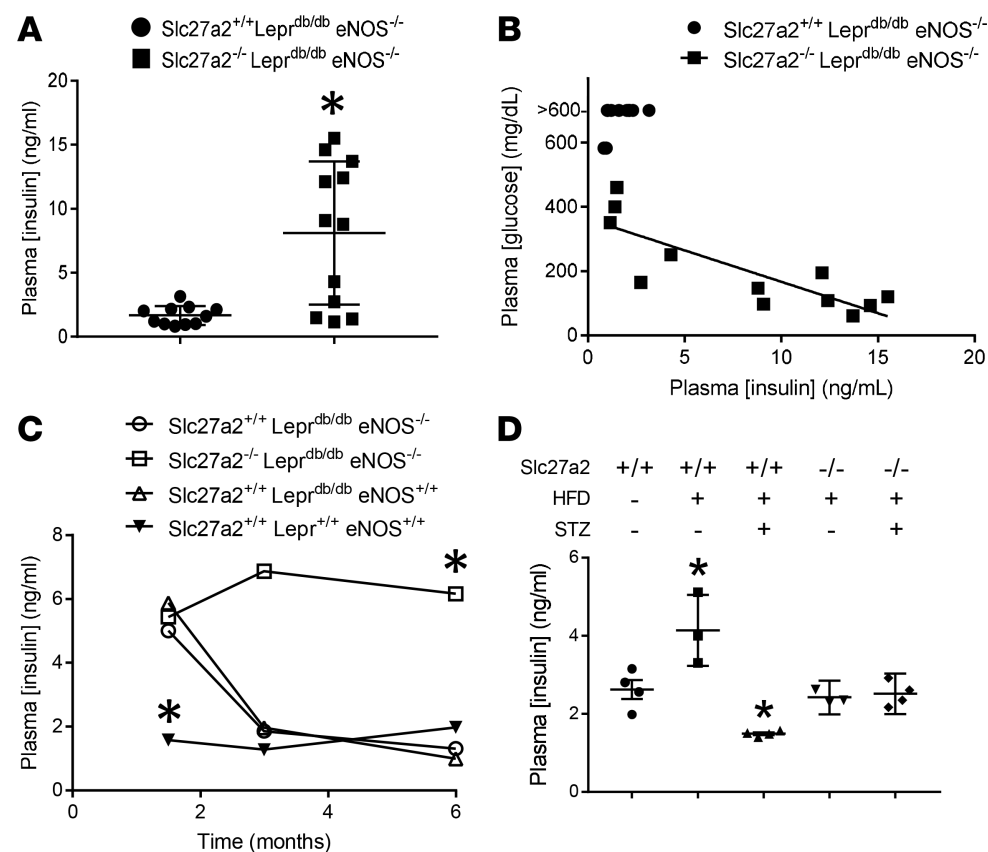

Figure 8. Plasma insulin levels are preserved in mice with FATP2 gene deletion. (A) Plasma insulin was measured by ELISA, as described in Methods, in DKD mice with or without FATP2 gene deletion. ${ }^{*} P=0.001$ by unpaired $t$ test. (B) Relationship between plasma glucose and corresponding insulin concentrations. $\mathrm{R}^{2}=0.682, P=0.0009$ by linear regression and Pearson's correlation. (C) Effect of FATP2 gene deletion on mean plasma insulin concentration over time. $n=4-6$ samples per group per time point. Error bars were omitted for clarity. ${ }^{*} P<0.02$ compared with other groups by ANOVA and Tukey's test for multiple comparisons. (D) Insulin levels in 6-month-old WT and Slc27a2 $2^{-1-}$ mice treated with or without HFD with or without low-dose STZ. ${ }^{*} P<0.05$ compared with other groups by ANOVA and Tukey's test for multiple comparisons. FATP2, fatty acid transport protein-2; DKD, diabetic kidney disease; STZ, streptozotocin.

DKD pathophysiology by reabsorbing large quantities of albumin-bound, filtered fatty acids, leading to lipotoxicity and apoptosis. However, approximately half of apical fatty acid uptake is attributable to other transport mechanisms (21), suggesting that other FATPs may also contribute to kidney lipotoxicity. We showed that multiple fatty acid transporters are expressed in the proximal tubule. Although this is consistent with fatty acids serving as a major substrate for ATP generation in metabolically demanding proximal tubules (41, 42), like FATP2, some transporters may be co-opted to transmit pathobiologic signals. Among candidates that might be additive to FATP2-induced lipotoxicity, FATP1 and FATP5 have been implicated in glucose intolerance and hepatosteatosis $(43,44)$. The $G$ protein-coupled fatty acid receptors GP40 (also known as FFA1) and GP84 (also known as FFA4) are expressed in renal tubular epithelial cell lines (45) and most prominently in the glomerular epithelial cell (podocyte) in vivo (21). GP40- and GP84-knockout mice were more susceptible and resistant, respectively, to nondiabetic models of kidney fibrosis (45). Lepr ${ }^{\mathrm{db} / \mathrm{db}} e \mathrm{NOS}^{-1-}$ mice treated with a combined GP40 activator and GP84 inhibitor demonstrated improved mortality, renal histology, GFR, and glycemic control $(31,45)$, similar to results we observed with FATP2 gene deletion. On the other hand, tubule-specific CD36-transgenic mice demonstrated no susceptibility to DKD, suggesting that downstream fatty acid channeling and metabolism may be transporter-specific (33).

The use of global knockout mice, to define the role of FATP2 in DKD, permitted the assessment of extrarenal FATP2 in DKD pathogenesis and potentially simulates the effect of a systemically administered FATP2 inhibitor. The most striking finding was markedly improved glycemic control in diabetic

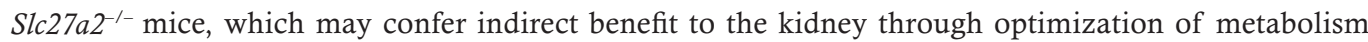

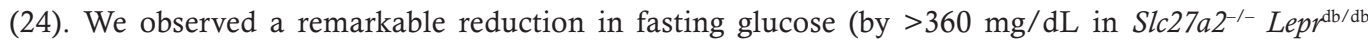

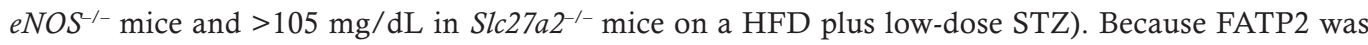

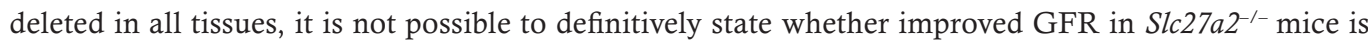
due to improved glycemic control or absence of proximal tubule FATP2-dependent fatty acid uptake and lipotoxicity. However, the lack of significant correlation between plasma glucose and GFR in the genetic or inducible models of DKD, as well as the divergent relationship between glucose and GFR in the 2 models (Supplemental Figure 10), suggest that glycemia is not the sole factor. 

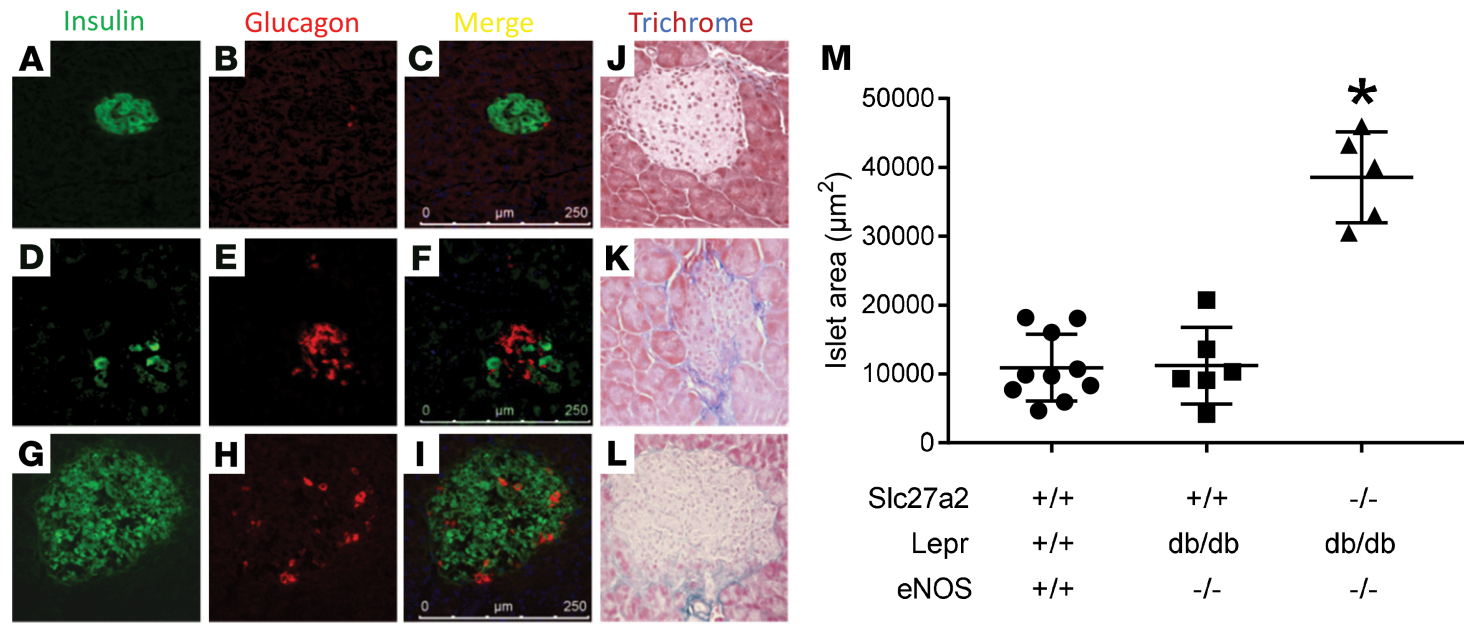

Figure 9. FATP2 deletion causes pancreatic islet hypertrophy. (A-I) Mouse pancreas frozen sections were labeled for $\beta$ cells with insulin antibodies and $\alpha$ cells with glucagon antibodies. (J-L) Mouse pancreas paraffin sections were stained with Masson's trichrome reagent. (A-C and J) WT mice.

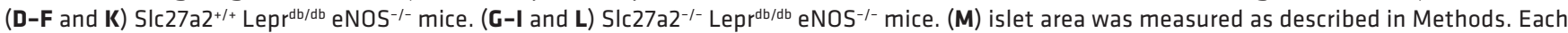
symbol represents mean area of all islets from one section per mouse. FATP2, fatty acid transport protein-2. ${ }^{*} P<0.01$ compared with other groups by ANOVA and Tukey's test for multiple comparisons.

Previous studies have shown that FATP2 shRNA administration to mice on a HFD improved mild hyperglycemia, as well as hepatosteatosis (23). In contrast to this report, we observed no significant effect of FATP2 deletion on hepatosteatosis, perhaps reflecting the relatively modest liver disease in Slc27a2 $2^{+/+}$ $L e p r^{\mathrm{db} / \mathrm{db}} e N O S^{-/-}$mice, differences in genetic background, and/or differences between acute shRNA knock-

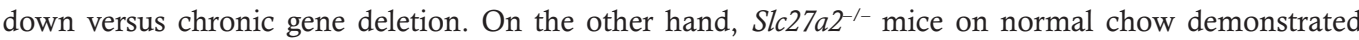
increased liver fatty acid content and lipid droplets (46), which is consistent with our data (Supplemental Figure 11B). Of other possible extrarenal FATP2 effects, a recent report demonstrates that FATP2-dependent arachidonic acid uptake activates a subset of neutrophils involved in cancer pathophysiology (47). The relevance of this intriguing pathway to DKD has not been studied, although inflammation and immune-mediated mechanisms have been implicated in DKD pathophysiology $(27,48)$.

The pathogenesis of type 1 and type 2 diabetes differs, but a final common pathway is loss of $\beta$ cells and insulin secretion. There are no available therapies that preserve $\beta$ cell function. Improved glycemia in $S l c 27 a 2^{-/-} \mathrm{Lepr}^{\mathrm{db} / \mathrm{db}} \mathrm{eNOS}^{-/-}$mice was achieved through $\beta$ cell hyperplasia and islet hypertrophy, resulting in sustained insulin levels and obesity. Islet hypertrophy and preserved insulin secretion

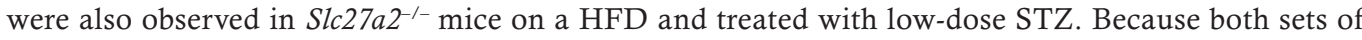
mice were sacrificed at 6 months, we cannot conclude whether FATP2 gene deletion prevents or merely delays hyperglycemia and DKD. Current diabetes investigation is intensely focused on strategies to replenish $\beta$ cells, including identification of the optimum cell source (stem cells vs. existing $\beta$ cells) (49-51) as well as factors that stimulate $\beta$ cell proliferation (52). The striking islet hypertrophy and $\beta$

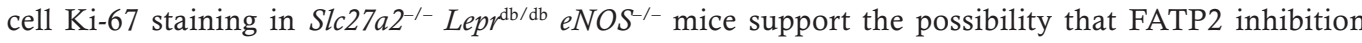
stimulates proliferation of existing $\beta$ cells and is consistent with pancreatic lipotoxicity to $\beta$ cells in the pathophysiology of type 2 diabetes (53). Single-cell RNA-seq studies have identified low FATP2 transcript copy number in $\beta$ cells (54), but FATP2 protein was not detected in $\beta$ cells from normal or diabetic pancreas by IHC (S. Khan and J.R. Schelling, unpublished observations), indicating that the FATP2 effect on $\beta$ cells may be indirect. The mechanism by which FATP2 gene deletion prevents insulin insufficiency will require further investigation.

In addition to faithfully mimicking progressive $\operatorname{DKD}(12,13), S_{c 27 a 2^{+/+}} \mathrm{Lepr}^{\mathrm{db} / \mathrm{db}} e N O S^{-/-}$mice experience premature mortality, which is also an important hallmark of DKD in humans (55). The normal

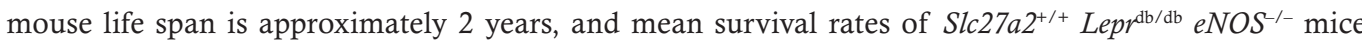
have ranged from 24 to 36 weeks $(26,31,32)$, with $100 \%$ mortality as early as 26 weeks (31). These data are consistent with the $85 \%$ mortality we observed at 26 weeks. Mortality was significantly attenuated

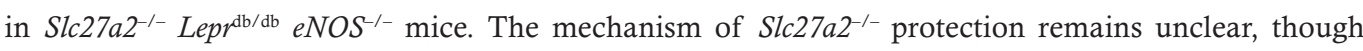
improved renal function and reduced glucotoxicity are undoubtedly factors. 


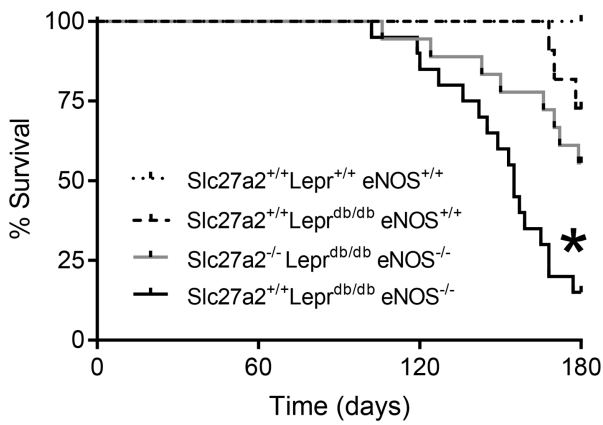

Figure 10. Attenuated mortality in SIc27a2 $2^{-/-}$Lepr $^{\mathrm{db} / \mathrm{db}} \mathrm{eNOS}^{-/-}$ mice. Kaplan-Meier survival curves. Results were derived from Slc27a2 $2^{+/+}$Lepr $^{+/+}$eNOS $^{+/+}(n=8)$, Slc27a2 $2^{+/+}$Lepr $^{\mathrm{db} / d \mathrm{db}}$ eNOS $^{+/+}(n$ $=11)$, SIc27a $2^{+/+}$Lepr $^{\mathrm{db} / d \mathrm{db}}$ eNOS $^{-/-}(n=20)$, and SIc27a2 $2^{-/-}$Lepr $^{\mathrm{db} / \mathrm{db}}$ eNOS ${ }^{-1-}(n=18)$ mice. ${ }^{*} P<0.001$ by $\chi^{2}$ and Mantel-Cox log-rank test compared with other groups.

Diabetes has become a worldwide epidemic and DKD is one of the most serious end-organ complications. In this report, we show that global deletion of the FATP2 gene confers a substantial kidney function benefit in heterogeneous murine models of progressive DKD. Although improved glycemic control in FATP2-knockout mice was anticipated, the magnitude of glucose reduction and the proportion of mice that achieved euglycemia, despite persistent hyperphagia and obesity, was unexpected. However, because glycemic control alone does not cure DKD (7), we speculate that FATP2 gene deletion improved renal function from a combination of direct proximal tubule and indirect metabolic effects, and establishing the relative contributions will require further investigation. There have been few significant DKD innovations over the past 40 years, but because of the impressive effects of FATP2 gene deletion on glycemic control and DKD progression, we are cautiously optimistic that FATP2 represents a promising, druggable target for the prevention of diabetes and its complications (56).

\section{Methods}

Animals. BKS.Cg-Leprdb Nos3tm1Unc/RhrsJ (Lepr $\left.{ }^{d b / d b} e N O S^{-/}\right)$and 129S-Slc27a2tm1Kds/J (Slc27a2 ${ }^{-/}$)

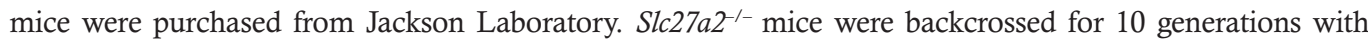

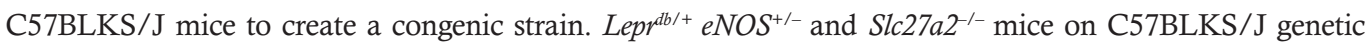
backgrounds were intercrossed to generate experimental groups. Mice were genotyped by PCR, as shown in Supplemental Figure 1. Age-matched male mice were slightly bigger than females (Figure 4A), but no gender differences were noted in any other experiments, consistent with observations by others in Lepr $^{d b / d b}$ eNOS $^{-/-}$ mice (57). Combined data from male and female mice are therefore presented for all other experiments.

Methods to induce type 2 diabetes and DKD were modified from refs. 58 and 59. Mice were fed a HFD (Teklad TD.06414, 60.3\% fat, 21.3\% carbohydrate, 18.4\% protein; Harlan Laboratories) for 6 months. At 3 months of age, mice were administered i.p. STZ ( $65 \mu \mathrm{g} / \mathrm{g}$ daily for 3 days). Diabetes was defined by fasting glucose greater than $200 \mathrm{mg} / \mathrm{dL}$.

Histology. Kidneys fixed in 4\% paraformaldehyde were embedded in paraffin blocks, and $10 \mu \mathrm{m}$ microtome sections were stained with Masson's trichrome, according to the guidelines from MilliporeSigma (HT15), as previously described (60). To assess for tubular atrophy and interstitial fibrosis, quantitative histomorphometry was conducted on images (original magnification, $\times 400$ ) that were overlaid with a $16 \times 22$ grid within Adobe Photoshop, as previously described (13). Coincidence of intersecting grid lines with tubule (nucleus, cytoplasm, or brush border) or Masson's trichrome-stained interstitium was counted; glomeruli, tubule lumens and blood vessels were omitted from calculations. The total of tubule cells plus fibrosed interstitium was defined as 100. Cytoplasmic lipid droplets were labeled with Oil Red O ( $0.5 \%$ in isopropanol, 5 minutes, room temperature), followed by hematoxylin counterstaining, as previously described (13). Stained sections were viewed by light microscopy (original magnification, $\times 400$ ). Glomerulosclerosis was scored using ImageJ software (NIH). Briefly, glomeruli were identified from Masson's trichrome-stained sections, with total glomerular area excluding Bowman's space and parietal epithelial cells. Thresholds were established to identify only blue-stained extracellular matrix. Data are expressed as the ratio of matrix/total glomerular area. Liver hepatosteatosis was scored from hematoxylin and eosin-stained sections according to methods adapted for mouse (61). The percentage of liver area that comprised lipid droplets was calculated using ImageJ software (NIH). Apoptosis was assessed by TUNEL assays, and results were quantified, as previously described (62).

IHC. Kidney IHC methods have been previously described in detail (60). FATP2 was labeled with rabbit anti-FATP2 IgG (GTX115526, GeneTex; 1:50, 16 hours, $\left.4^{\circ} \mathrm{C}\right)$. Proximal tubules were counterstained with goat anti-Glut5 IgG (SC-14844, Santa Cruz Biotechnology; 1:200, 16 hours, $4^{\circ} \mathrm{C}$ ) or mouse 
anti- $\gamma$-glutamyltransferase-1 IgG (ST1551, Calbiochem; 1:200, 16 hours, $4^{\circ} \mathrm{C}$ ). Macrophages were labeled with rat F4/80 IgG (CL8940AP, Cedarlane; 1:200, 16 hours, $4^{\circ} \mathrm{C}$ ) or rabbit anti-CSF-1 receptor (TA300025, Origene; 1:50, 16 hours, $4^{\circ} \mathrm{C}$ ) from 4\% paraformaldehyde-fixed paraffin sections and quantified from 4 random fields per section. Secondary Alexa Fluor 488 and 568 antibodies were used (Invitrogen Life technologies, 1:200, room temperature, 1 hour).

Mouse pancreas was frozen at $-80^{\circ} \mathrm{C}$. Samples were sectioned to $5 \mu \mathrm{m}$ by cryostat, fixed in paraformaldehyde (4\%, 10 minutes, room temperature), rinsed in PBS, and permeabilized with Triton X-100 (MilliporeSigma; $0.2 \%$ in PBS, 10 minutes, room temperature). Sections were blocked with serum (5\% in PBS, 1 hour, room temperature). Antibodies were AF488-conjugated rabbit anti-insulin IgG (9016, Cell Signaling; $1: 200,16$ hours, $4^{\circ} \mathrm{C}$ ), eFluor 570-conjugated mouse monoclonal anti-glucagon IgG (ICACLS, Invitrogen Thermo Fisher Scientific; $10 \mu \mathrm{g} / \mathrm{mL}, 16$ hours, $4^{\circ} \mathrm{C}$ ), and AF594-conjugated rat anti-Ki-67 IgG (151214, BioLegend; 1:100, 16 hours, $\left.4^{\circ} \mathrm{C}\right)$. Sections were mounted in SlowFade Diamond Antifade Mountant with DAPI (Invitrogen) and viewed by confocal microscopy (Leica).

Immunoblot analysis. Immunoblot methods have been previously described (63). Briefly, samples (20 $\mu$ g protein per lane) were resolved by SDS-PAGE and transferred to polyvinylidene difluoride membranes. Blots were blocked in 5\% nonfat dried milk and probed with anti- $\alpha$-smooth muscle actin (A2547, MilliporeSigma; 1:1000, 16 hours, $4^{\circ} \mathrm{C}$ ) or anti-HSP47 (SC-8352, Santa Cruz; 1:1000, overnight, $4^{\circ} \mathrm{C}$ ) IgG and then HRP-conjugated $\operatorname{IgG}(1: 10,000,1$ hour, room temperature). Band intensity was detected by enhanced chemiluminescence. Blots were exposed to stripping buffer (Thermo Scientific; 10 minutes, room temperature) and then reprobed with anti-GAPDH IgG (2118, Cell Signaling; 1:3,000, 1 hour, room temperature) as a loading control.

GFR measurement. FITC-labeled inulin clearance was measured in mice as previously described (64), with minor modifications. FITC-inulin ( $5 \%$ in PBS) was dialyzed against $0.9 \%$ saline overnight using a $1.0 \mathrm{kDa}$ cutoff dialysis membrane (Spectra/Pro 6, Spectrum Labs) to remove residual FITC not bound to inulin. FITC-inulin $(3.74 \mu \mathrm{L} / \mathrm{g}$ body weight) was injected into the retro-orbital vein of mice that were anesthetized with isoflurane. Blood samples were collected in heparinized capillary tubes from the tail vein at 3 , $7,10,15,35,55$, and 75 minutes after the FITC-inulin injection. Plasma samples were centrifuged, loaded into black-walled, 96-well microplates (Greiner Bio-One). Fluorescence readings were measured at $485 \mathrm{~nm}$ excitation/538 nm emission using an EnSpire 2300 Multilabel plate reader (PerkinElmer). Fluorescence values were fitted to a 2-phase exponential decay curve using nonlinear regression (GraphPad Prism 7), and GFR was calculated as previously described (65).

Albuminuria measurement. Spot urine samples were obtained from conscious mice in afternoons and at the time of sacrifice. Urine albumin was assayed by ELISA according to the manufacturer instructions (Albuwell M, Exocell). Urine creatinine concentration was measured using a picric acid method according to manufacturer instructions (Creatinine Companion, Exocell)

Reverse transcriptase PCR. Total RNA was extracted from whole mouse organs using RNeasy Mini Kit (QIAGEN) in accordance with the protocol described by the manufacturer. RNA concentrations were determined using the NanoDrop 2000 Spectrophotometer (Thermo Scientific). Reverse transcription (RT) was performed using $5 \mu \mathrm{g}$ total RNA, which was processed with the SuperScript III First-Strand Synthesis System for RT-PCR kit (Invitrogen) according to the manufacturer's protocols. PCR reactions were conducted in $20 \mu \mathrm{l}$ volume using EmeraldAmp Max PCR Master Mix 2X premix (Takara Bio Inc.) according to recommended protocol and PCR cycling conditions. PCR products underwent $2 \%$ agarose gel electrophoresis and bands were identified by ethidium bromide staining and photographed. FATP2 primer sequences were 5'-TGTGCCAGGTCATGAGGGTCG-3' (forward) and 5'-CTCAATGGTATCTTGTATCCTCAGGAA-3' (reverse), yielding a 151 bp amplicon. GAPDH primer sequences were 5'-GTGGAAGGGCTCATGACCACAG-3' (forward) and 5'-ATACTTGGCAGGTTTCTCCAGGC-3' (reverse), yielding a 255 bp product.

Quantitative RT-PCR was conducted as follows. Total RNA (10 ng per well) was reverse-transcribed as described above, and cDNA was QPCR-amplified using the Applied Biosystems TaqMan Gene Expression System. cDNA samples were diluted to a concentration of $10 \mathrm{ng} / 9 \mu \mathrm{L}$ and aliquoted into 2 wells, to which $10 \mu \mathrm{L}$ of TaqMan Gene Expression Master Mix and $1 \mu \mathrm{L}$ of either human FATP isoform or human GAP$\mathrm{DH}$ primers was added. Analysis was performed on an Applied Biosystems 7500 Real-time PCR System running 7500 Real Time Software v2.3.

Blood glucose assays. Blood samples $(2-3 \mu \mathrm{l})$ were obtained by tail nick with a 25 -gauge needle in unrestrained mice maintained in a quiet environment for 5 minutes. Blood glucose was measured in mice between the ages of 6 weeks to 6 months using a TRUE METRIX glucometer (Trividia). 
Food consumption. Food intake was determined in 3- to 4-month-old mice by weighing chow daily for 1 week, and recording daily differences as chow consumption in $\mathrm{g} / \mathrm{d}$.

Physical activity tracking. A solitary mouse in one cage was removed from the animal care facility. A 5 $\mathrm{mm}$ adhesive cursor was placed on the posterior neck, and the mouse remained undisturbed for 10 minutes. Mouse activity was then recorded with a Logitech C270 webcam attached to an HP ProBook 4540s laptop computer, and the cursor distance traveled was analyzed by Tracker 5.1.1 (Douglas Brown, Open Source Physics, https://www.compadre.org/osp) software. Recordings were made for three 10-minute intervals, and data are expressed as the mean from the triplicate recordings.

Plasma insulin assays. Plasma insulin was measured as previously described (66) using a mouse insulin ELISA kit according to manufacturer instructions (Crystal Chem).

Statistics. Graphical data are presented as mean \pm SEM. Data from multiple groups were statistically analyzed by 1-way ANOVA and Tukey's test for multiple testing of nonparametric data (GraphPad Prism 7). Data from 2 groups were analyzed by unpaired 2-tailed $t$ test (GraphPad Prism 7). The relationship between phenotypes and Slc27a2 null allele number was determined by linear regression using GraphPad Prism 7. Statistical significance for all analyses is defined as a $P$ value of less than 0.05 . For statistical analyses of plasma glucose, readings exceeding the maximum threshold of the glucometer $(600 \mathrm{mg} / \mathrm{dL})$ were arbitrarily assigned a value of $700 \mathrm{mg} / \mathrm{dL}$ but graphed as greater than $600 \mathrm{mg} / \mathrm{dL}$.

Study approval. All protocols and procedures were approved by the Institutional Animal Care and Use Committee of Case Western Reserve University and conducted in accordance with the Guide for the Care and Use of Laboratory Animals (National Academies Press, 2011).

\section{Author contributions}

SK designed research studies, conducted experiments, acquired, and analyzed data. RG designed research studies, conducted experiments, acquired, and analyzed data. CA conducted experiments, acquired, and analyzed data. MB designed research studies and analyzed data. JC designed research studies and analyzed data. JS designed research studies, acquired, and analyzed data.

\section{Acknowledgments}

This work was supported by NIH grants R01 DK067528 and U01 DK106965 (to JRS). We are grateful to Susan Gurley (Division of Nephrology, University of Oregon Health Science Center) for assistance with establishing the FITC-inulin GFR assays, to Yisheng Yang (Department of Medicine, Division of Endocrinology, MetroHealth Campus, Case Western Reserve University) for assistance with insulin assays and review of the manuscript, and to Jeff Garvin (Department of Physiology and Biophysics, Case Western Reserve University) for helpful discussions and careful review of the manuscript.

Address correspondence to: Jeffrey R. Schelling, MetroHealth Medical Center, 2500 MetroHealth Drive, Rammelkamp R425, Cleveland, Ohio 44019-1998, USA. Phone: 216.778.8806; Email: jeffrey.schelling@case.edu.

1. [No authors listed]. USRDS 2018 Annual Data Report. United States Renal Data System. https://www.usrds.org/adr.aspx. Accessed June 29, 2020.

2. Lewis EJ, Hunsicker LG, Bain RP, Rohde RD. The effect of angiotensin-converting-enzyme inhibition on diabetic nephropathy. The Collaborative Study Group. N Engl J Med. 1993;329(20):1456-1462.

3. Brenner BM, et al. Effects of losartan on renal and cardiovascular outcomes in patients with type 2 diabetes and nephropathy. NEngl J Med. 2001;345(12):861-869.

4. Mann JFE, et al. Liraglutide and renal outcomes in type 2 diabetes. N Engl J Med. 2017;377(9):839-848

5. Wanner C, et al. Empagliflozin and progression of kidney disease in type 2 diabetes. N Engl J Med. 2016;375(4):323-334.

6. Perkovic V, et al. Canagliflozin and renal outcomes in type 2 diabetes and nephropathy. N Engl J Med. 2019;380(24):2295-2306.

7. Newman DJ, et al. Systematic review on urine albumin testing for early detection of diabetic complications. Health Technol Assess. 2005;9(30):iii-vi, xiii.

8. Schelling JR. Tubular atrophy in the pathogenesis of chronic kidney disease progression. Pediatr Nephrol. 2016;31(5):693-706.

9. Brosius FC, et al. Mouse models of diabetic nephropathy. J Am Soc Nephrol. 2009;20(12):2503-2512.

10. Rodriguez WE, et al. Pioglitazone mitigates renal glomerular vascular changes in high-fat, high-calorie-induced type 2 diabetes mellitus. Am J Physiol Renal Physiol. 2006;291(3):F694-F701.

11. Tsai HC, et al. Elafibranor inhibits chronic kidney disease progression in NASH mice. Biomed Res Int. 2019;2019:6740616.

12. Zhao HJ, et al. Endothelial nitric oxide synthase deficiency produces accelerated nephropathy in diabetic mice. $J A m$ Soc Nephrol. 2006;17(10):2664-2669.

13. Khan S, et al. Lipotoxic disruption of NHE1 interaction with PI(4,5)P2 expedites proximal tubule apoptosis. J Clin Invest. 
2014;124(3):1057-1068

14. Moorhead JF, Chan MK, El-Nahas M, Varghese Z. Lipid nephrotoxicity in chronic progressive glomerular and tubulo-interstitial disease. Lancet. 1982;2(8311):1309-1311.

15. Weinberg JM. Lipotoxicity. Kidney Int. 2006;70(9):1560-1566.

16. Proctor G, Jiang T, Iwahashi M, Wang Z, Li J, Levi M. Regulation of renal fatty acid and cholesterol metabolism, inflammation, and fibrosis in Akita and OVE26 mice with type 1 diabetes. Diabetes. 2006;55(9):2502-2509.

17. Kamijo A, et al. Urinary free fatty acids bound to albumin aggravate tubulointerstitial damage. Kidney Int. 2002;62(5):1628-1637.

18. Arici M, Chana R, Lewington A, Brown J, Brunskill NJ. Stimulation of proximal tubular cell apoptosis by albumin-bound fatty acids mediated by peroxisome proliferator activated receptor-gamma. J Am Soc Nephrol. 2003;14(1):17-27.

19. Hirsch D, Stahl A, Lodish HF. A family of fatty acid transporters conserved from mycobacterium to man. Proc Natl Acad Sci USA. 1998;95(15):8625-8629.

20. Johnson AC, Stahl A, Zager RA. Triglyceride accumulation in injured renal tubular cells: alterations in both synthetic and catabolic pathways. Kidney Int. 2005;67(6):2196-2209.

21. Khan S, et al. Kidney proximal tubule lipoapoptosis is regulated by fatty acid transporter-2 (FATP2). J Am Soc Nephrol. 2018;29(1):81-91.

22. Park J, et al. Single-cell transcriptomics of the mouse kidney reveals potential cellular targets of kidney disease. Science. 2018;360(6390):758-763.

23. Falcon A, et al. FATP2 is a hepatic fatty acid transporter and peroxisomal very long-chain acyl-CoA synthetase. Am J Physiol Endocrinol Metab. 2010;299(3):E384-E393.

24. Reidy K, Kang HM, Hostetter T, Susztak K. Molecular mechanisms of diabetic kidney disease. J Clin Invest. 2014;124(6):2333-2340.

25. Heinzer AK, et al. A very long-chain acyl-CoA synthetase-deficient mouse and its relevance to X-linked adrenoleukodystrophy. Hum Mol Genet. 2003;12(10):1145-1154.

26. Mohan S, et al. Diabetic eNOS knockout mice develop distinct macro- and microvascular complications. Lab Invest. 2008;88(5):515-528.

27. Tesch GH. Diabetic nephropathy - is this an immune disorder? Clin Sci. 2017;131(16):2183-2199.

28. Iwano M, Plieth D, Danoff TM, Xue C, Okada H, Neilson EG. Evidence that fibroblasts derive from epithelium during tissue fibrosis. J Clin Invest. 2002;110(3):341-350.

29. Humphreys BD, et al. Fate tracing reveals the pericyte and not epithelial origin of myofibroblasts in kidney fibrosis. Am J Pathol. 2010;176(1):85-97.

30. Petersen MC, Vatner DF, Shulman GI. Regulation of hepatic glucose metabolism in health and disease. Nat Rev Endocrinol. 2017;13(10):572-587.

31. Li Y, et al. Fatty acid receptor modulator PBI-4050 inhibits kidney fibrosis and improves glycemic control. JCI Insight. 2018;3(10):120365.

32. Takahashi T, Harris RC. Role of endothelial nitric oxide synthase in diabetic nephropathy: lessons from diabetic eNOS knockout mice. J Diabetes Res. 2014;2014:590541

33. Kang HM, et al. Defective fatty acid oxidation in renal tubular epithelial cells has a key role in kidney fibrosis development. Nat Med. 2015;21(1):37-46.

34. Davis TM, et al. Effects of fenofibrate on renal function in patients with type 2 diabetes mellitus: the Fenofibrate Intervention and Event Lowering in Diabetes (FIELD) Study. Diabetologia. 2011;54(2):280-290.

35. ACCORD Study Group, et al. Effects of combination lipid therapy in type 2 diabetes mellitus. N Engl J Med. 2010;362(17):1563-1574.

36. Grgic I, et al. Targeted proximal tubule injury triggers interstitial fibrosis and glomerulosclerosis. Kidney Int. 2012;82(2):172-183.

37. Maluf DG, et al. Evaluation of molecular profiles in calcineurin inhibitor toxicity post-kidney transplant: input to chronic allograft dysfunction. Am J Transplant. 2014;14(5):1152-1163.

38. Cheng L, et al. Zoledronate dysregulates fatty acid metabolism in renal tubular epithelial cells to induce nephrotoxicity. Arch Toxicol. 2018;92(1):469-485.

39. Ahowesso C, et al. Chemical inhibition of fatty acid absorption and cellular uptake limits lipotoxic cell death. Biochem Pharmacol. 2015;98(1):167-181.

40. Mendez-Sanchez N, Cruz-Ramon VC, Ramirez-Perez OL, Hwang JP, Barranco-Fragoso B, Cordova-Gallardo J. New aspects of lipotoxicity in nonalcoholic steatohepatitis. Int J Mol Sci. 2018;19(7):E2034.

41. Guder WG, Wagner S, Wirthensohn G. Metabolic fuels along the nephron: pathways and intracellular mechanisms of interaction. Kidney Int. 1986;29(1):41-45.

42. Balaban RS, Mandel LJ. Metabolic substrate utilization by rabbit proximal tubule. An NADH fluorescence study. Am J Physiol. 1988;254(3 Pt 2):F407-F416.

43. Kim JK, et al. Inactivation of fatty acid transport protein 1 prevents fat-induced insulin resistance in skeletal muscle. J Clin Invest. 2004;113(5):756-763.

44. Doege $\mathrm{H}$, et al. Silencing of hepatic fatty acid transporter protein 5 in vivo reverses diet-induced non-alcoholic fatty liver disease and improves hyperglycemia. J Biol Chem. 2008;283(32):22186-22192.

45. Gagnon L, et al. A newly discovered antifibrotic pathway regulated by two fatty acid receptors: GPR40 and GPR84. Am J Pathol. 2018;188(5):1132-1148

46. Perez VM, Gabell J, Behrens M, Wase N, DiRusso CC, Black PN. Deletion of fatty acid transport protein 2 (FATP2) in the mouse liver changes the metabolic landscape by increasing the expression of PPAR $\alpha$-regulated genes. J Biol Chem. 2020;295(17):5737-5750.

47. Veglia F, et al. Fatty acid transport protein 2 reprograms neutrophils in cancer. Nature. 2019;569(7754):73-78.

48. Matoba K, Takeda Y, Nagai Y, Kawanami D, Utsunomiya K, Nishimura R. Unraveling the role of inflammation in the pathogenesis of diabetic kidney disease. Int J Mol Sci. 2019;20(14):E3393.

49. Pagliuca FW, et al. Generation of functional human pancreatic $\beta$ cells in vitro. Cell. 2014;159(2):428-439.

50. Rezania A, et al. Reversal of diabetes with insulin-producing cells derived in vitro from human pluripotent stem cells. Nat Bio- 
technol. 2014;32(11):1121-1133.

51. Dor Y, Brown J, Martinez OI, Melton DA. Adult pancreatic beta-cells are formed by self-duplication rather than stem-cell differentiation. Nature. 2004;429(6987):41-46.

52. Moullé VS, Ghislain J, Poitout V. Nutrient regulation of pancreatic $\beta$-cell proliferation. Biochimie. 2017;143:10-17.

53. Oh YS, Bae GD, Baek DJ, Park EY, Jun HS. Fatty acid-induced lipotoxicity in pancreatic $\beta$-cells during development of type 2 diabetes. Front Endocrinol (Lausanne). 2018;9(1):384

54. Li J, et al. Single-cell transcriptomes reveal characteristic features of human pancreatic islet cell types. EMBO Rep. 2016;17(2):178-187.

55. Afkarian M, et al. Kidney disease and increased mortality risk in type 2 diabetes. J Am Soc Nephrol. 2013;24(2):302-308

56. Black PN, Ahowesso C, Montefusco D, Saini N, DiRusso CC. Fatty acid transport proteins: targeting FATP2 as a gatekeeper involved in the transport of exogenous fatty acids. Medchemcomm. 2016;7(4):612-622.

57. Ma Y, et al. Comparison of diabetic nephropathy between male and female eNOS ${ }^{-/-} d b / d b$ mice. Am J Physiol Renal Physiol. 2019;316(5):F889-F897.

58. Lo L, et al. Diabetes is a progression factor for hepatic fibrosis in a high fat fed mouse obesity model of non-alcoholic steatohep atitis. J Hepatol. 2011;55(2):435-444.

59. Glastras SJ, et al. Mouse models of diabetes, obesity and related kidney disease. PLoS One. 2016;11(8):e0162131.

60. Khan S, et al. Mesangial cell integrin $\alpha v \beta 8$ provides glomerular endothelial cell cytoprotection by sequestering TGF- $\beta$ and regulating PECAM-1. Am J Pathol. 2011;178(2):609-620.

61. Liang W, et al. Establishment of a general NAFLD scoring system for rodent models and comparison to human liver pathology. PLoS One. 2014;9(12):e115922.

62. Wu KL, et al. Renal tubular epithelial cell apoptosis is associated with caspase cleavage of the $\mathrm{NHE}^{\mathrm{N}} \mathrm{Na}^{+} / \mathrm{H}^{+}$exchanger. $A m J$ Physiol Renal Physiol. 2003;284(4):F829-F839.

63. Khan S, et al. Lipotoxic disruption of NHE1 interaction with PI(4,5)P2 expedites proximal tubule apoptosis. J Clin Invest. 2014;124(3):1057-1068.

64. Gurley SB, et al. Influence of genetic background on albuminuria and kidney injury in Ins2 ${ }^{+/ C 96 Y}$ (Akita) mice. Am J Physiol Renal Physiol. 2010;298(3):F788-F795.

65. Qi Z, et al. Characterization of susceptibility of inbred mouse strains to diabetic nephropathy. Diabetes. 2005;54(9):2628-2637.

66. Yang Y, et al. The Krüppel-like zinc finger protein GLIS3 transactivates neurogenin 3 for proper fetal pancreatic islet differentiation in mice. Diabetologia. 2011;54(10):2595-2605. 